\title{
A Role for Receptor-Interacting Protein Kinase-1 in Neutrophil Extracellular Trap Formation in Patients with Systemic Lupus Erythematosus: a Preliminary Study
}

\author{
Ruru Guo Yang Tu $^{\mathrm{a}}$ Shaowei Xie ${ }^{\mathrm{b}} \quad$ Xue song Liu ${ }^{\mathrm{b}} \quad$ Yang Song $^{\mathrm{a}}$ Suli Wang \\ Xiaoxiang Chen ${ }^{\mathrm{a}}$ Liangjing $\mathrm{Lu}^{\mathrm{a}}$ \\ aDepartment of Rheumatology, Renji Hospital, School of Medicine, Shanghai Jiaotong University, \\ Shanghai, bDepartment of Ultrasound, Renji Hospital, School of Medicine, Shanghai Jiaotong University, \\ Shanghai, P. R. China
}

\section{Key Words}

Receptor-interacting protein kinase-1 • Neutrophil extracellular trap • Necroptosis • Systemic lupus erythematosus

\begin{abstract}
Background/Aims: Neutrophil extracellular traps (NETs) are known to play an important role in systemic lupus erythematosus (SLE) by triggering innate and adaptive immune responses. The molecular mechanisms responsible for their formation in SLE are still unclear. In this study, we aim to characterize the role of the receptor-interacting protein kinase-1 (RIPK1), a homologous serine/threonine kinase previously implicated in the regulation of necroptosis and tissue injury, in decreasing neutrophil death and formation of NETs, and to investigate the clinical implications of RIPK1 in SLE. Methods: Patients with SLE $(n=50)$ and healthy donors $(n=35)$ were enrolled in in vitro studies. Management of SLE patients was evaluated using the SLE disease activity index 2000 (SLEDAI-2K) score and laboratory variables. The mRNA level of RIPKs was measured by quantitative polymerase chain reaction (qPCR). Intracellular RIPK1 and RIPK3 production by peripheral blood leukocytes was detected by four-color flow cytometry and confirmed by automatic western blotting. TNF- $\alpha$, IFN- $\gamma$, IL-1 $\beta$, IL-2, IL-8, IL-18, and RIPK1 were measured by enzyme-linked immunosorbent assay. Cell death was assayed by Sytox green dye from peripheral neutrophils stimulated by RIPK-1-stabilizer necrostatin-1 (nec-1) and phorbol 12-myristate 13-acetate (PMA). Immunofluorescence staining and confocal microscopy were used to detect NET formation ex vivo. Quantification of NETs was determined by fluorescence spectrometry. Results: IFN- $\gamma$, IL-1 $\beta$, IL- 8 , and IL-18 levels in serum were increased in SLE patients compared to controls. However, the expression of TNF- $\alpha$, IL-2, and RIPK1 were decreased. In addition, we observed significant differences in the expression of RIPK1 in peripheral blood leukocytes. Of all the leukocytes, RIPK1 expression was significantly lower in neutrophils. Furthermore, we studied NETs formation in neutrophils of SLE with decreased RIPK1 expression, and these show increased susceptibility to NETosis,
\end{abstract}

Liangjing Lu

and Xiaoxiang Chen

KARGER
Department of Rheumatology, Renji Hospital, School of Medicine, Shanghai Jiaotong

University, 145 Middle Shandong Rd, Shanghai 200001, (P. R. China)

Tel. +86 18817821418, E-Mail lu_liangjing@163.com; xiaoxiang0721@126.com 


\section{Cellular Physiology Cell Physiol Biochem 2018;45:2317-2328 \\ and Biochemistry Published onlIne: IVarch 16, 2018 \begin{tabular}{l|l} 
DOI: 10.1159/000488179 & $\begin{array}{l}\text { C } 2018 \text { The Author(s). Published by S. Karger AG, Basel } \\
\text { www.karger.com/cpb }\end{array}$
\end{tabular} \\ Guo et al.: RIPK1 Involved in Nets Formation in SLE}

when stimulated with PMA and/or nec-1. Importantly, RIPK1 expression in neutrophils negatively correlated with ESR, CRP, 24-hour urine total protein, and the disease activity index in SLE. Conclusion: These data represent the first report of decreased RIPK1 expression in neutrophils of SLE patients and imply that RIPK1 may be involved in neutrophil death and NET formation. We suggest that RIPK1 is a potential biomarker to predict disease activity.

(C) 2018 The Author(s)

Published by S. Karger AG, Basel

\section{Introduction}

Systemic lupus erythematosus (SLE) is a multifactorial autoimmune disease, which is characterized by the loss of tolerance to nuclear antigens, the deposition of immune complexes in tissues, and multi organ involvement. SLE afflicts mostly women of childbearing age. Autoantibodies directed against nuclear antigens and neutrophil proteins form immune complexes in situ or deposits in kidneys of SLE patients, which indicates that the disease is "antigen driven". The timely removal of immune complexes may be crucial for general homeostasis and to avoid presentation of self-antigens. Most current researches focus on $\mathrm{B}$ and $\mathrm{T}$ lymphocytes and, to a lesser extent, dendritic cells, and their involvement in the pathogenesis of SLE [1]. However, the main source of self-antigens has been suggested to be apoptotic or necrotic material that SLE patients fail to dispose of, for example, NETs and the delayed clearance of NETosis remnants [2]. Evidence accrued over the past decade indicates that SLE patients have profound disruptions in innate immunity which could play a crucial part in the initiation and perpetuation of the disease $[1,3,4]$. Neutrophils are the most abundant leukocytes in the human body, however, they have a short lifespan and are continuously released from the bone marrow. As part of the innate immune system, recent studies have pushed the neutrophil to the forefront of the pathogenesis of SLE.

The discovery of neutrophil extracellular traps (NETs) revealed that neutrophils can immobilize and kill invading microbes by NET formation [1]. NETs are formed through a regulated cell death program (NETosis) [5] and consist of DNA structures covered with antimicrobial enzymes. Several proteins adhere to NETs [6, 7], including elastase and over 30 components of primary and secondary granules, among which are components with bactericidal activity. In some patients with SLE, the clearance of NETs is impaired and correlates with disease activity and renal involvement [8]. The residual and circulating NETs complexes contain degraded chromatin, which can serve as autoantigens, thus exacerbating the disease activity of SLE [2]. Furthermore, compelling evidence has emerged that NETs are involved in a wide variety of pathologies including infectious diseases, cancer, atherosclerosis, and autoimmune diseases, emphasizing the need to gain insight into the molecular mechanisms underlying this phenomenon [9]. Interestingly, a recent study using genetic and pharmacological approaches reported [10] that PMA-induced NETosis is inhibited by RIPK-1-stabilizer necrostatin-1 (nec-1), underscoring the necessity to revisit the potential involvement of the RIPKs in this cell death modality.

The most characterized form of regulated cell death is necroptosis. Although no unique marker of necroptosis exists, it is distinguished from other cell death modalities at the molecular level by its dependence on RIPK1, RIPK3, and its downstream effector MLKL (the mixed lineage kinase domain-like protein) [11]. However, accumulating data support that although RIPK1 and RIPK3 kinases first emerged as mediators of necroptosis, they also promote caspase-8-dependent apoptosis and proinflammatory gene expression [12-14]. RIPK1 and RIPK3 promote at least three distinct responses: necroptosis, apoptosis, and cell death-independent inflammation, and the loss of homeostasis of any of these pathways may contribute to the development of pathology $[12,15,16]$. Therefore, the aim of this study is to determine RIPK1 expression and investigate whether RIPK1 contributes to NETs formation in SLE patients. 


\section{Cellular Physiology Cell Physiol Biochem 2018;45:2317-2328 \begin{tabular}{ll|l} 
and Biochemistry Published onIIne: IVarch 16, 2018 & $\begin{array}{l}\text { (c) } 2018 \text { The Author(s). Published by S. Karger AG, Basel } \\
\text { www.karger.com/cpb }\end{array}$
\end{tabular}

\section{Materials and Methods}

\section{Subjects}

American College of Rheumatology criteria for SLE and SLEDAI-2K scores were adopted for diagnosis and disease activity assessment of SLE patients, respectively $[17,18]$. We enrolled age-and sex-matched SLE patients $(n=50)$ and healthy donors $(n=35)$ from the Department of Rheumatology, Shanghai Renji Hospital between October 2016 and May 2017. Serum samples were obtained from all 85 participants and stored at $-80^{\circ} \mathrm{C}$ prior to analysis. Detail clinical and related laboratory data were collected at the time serum samples were obtained. Exclusion criteria for this study included infectious diseases, leukemia, lymphoma, and other connective tissue diseases. The study was conducted according to the Declaration of Helsinki, and ethics committee approval for this study was obtained from the institutional review board of Renji Hospital.

\section{ELISAS}

Serum RIPK1 (n (SLE) = 20, n (CTL) = 15), TNF- $\alpha(\mathrm{n}(\mathrm{SLE})=20, \mathrm{n}(\mathrm{CTL})=20), \mathrm{IL}-2$ (n $(\mathrm{SLE})=44, \mathrm{n}$ $(\mathrm{CTL})=26), \mathrm{IFN}-\gamma(\mathrm{n}(\mathrm{SLE})=18, \mathrm{n}(\mathrm{CTL})=18), \mathrm{IL}-1 \beta(\mathrm{n}(\mathrm{SLE})=20, \mathrm{n}(\mathrm{CTL})=20), \mathrm{IL}-8(\mathrm{n}(\mathrm{SLE})=22, \mathrm{n}$ $(\mathrm{CTL})=19)$, and IL-18 (n (SLE) $=20, \mathrm{n}(\mathrm{CTL})=20$ ) were measured by a commercially available ELISA kit (R\&D Systems, Minneapolis, US). Although one sample of serum is not sufficient to complete all the cytokine ELISAs, one cytokine can be measured in at least in 15 samples. According to the suggested protocol, a microplate was coated with diluted capture antibody and incubated overnight at $4^{\circ} \mathrm{C}$. After washing three times with $0.05 \%$ Tween-20 in phosphate buffered saline (PBS), the plate was blocked with $1 \%$ bovine serum albumin (BSA) in PBS at room temperature for $2 \mathrm{~h}$. The plate was then washed three times, and diluted samples and standards were then added in duplicate to the corresponding wells for $2 \mathrm{~h}$ at room temperature. After washing, captured cytokines reacted with the detection antibody, and the plate was incubated $2 \mathrm{~h}$ at room temperature. Streptavidin-HRP was then added to each well for $20 \mathrm{~min}$ at room temperature. Color was developed by incubating with substrate solution for $20 \mathrm{~min}$ at room temperature. The reaction was terminated with stop solution, and absorbance was read using a microplate reader set to $450 \mathrm{~nm}$.

\section{Peripheral blood mononuclear cells isolation}

Five milliliters of whole blood was obtained from SLE patients and healthy donors using ACD tubes (BD Vacutainer). Peripheral blood mononuclear cells (PBMCs) were isolated by Polymorph Prep (Axis-Shield) according to the instructions recommended by the manufacturer.

\section{RNA isolation and quantitative polymerase chain reaction ( $q P C R$ )}

Total RNA from PBMCs was isolated using TRIzol according to the manufacturer's instruction (Invitrogen, USA) and stored at $-80^{\circ} \mathrm{C}$. The RNA concentration in each sample was determined by a NanoDrop 2000 Spectrophotometer. Oligonucleotides as specific primers for RIPK1 and RIPK3 were synthesized by a commercial laboratory (Sangon Biotech, China). As a PCR control reaction, $\beta$-actin was also measured in each run. Primer sequences are as follows (RIPK1, NM_003804; RIPK3, NM_006871). RIPK1, Forward: 5'-TATCCCAGTGCCTGAGACCAAC-3'; Reverse: 3'-GTAGGCTCCAATCTGAATGCCAG-5'. RIPK3, Forward: 5'-GCTACGATGTGGCGGTCAAGAT-3'; Reverse: 3'-TTGGTCCCAGTTCACCTTCTCG-5'.

An equal amount of total RNA from each individual sample was used as a template in cDNA preparation with Superscript II (Invitrogen). cDNA amplification was performed using the Brilliant SYBR Green QPCR Master Mix kit (Stratagene, La Jolla, CA) according to the instructions recommended by the manufacturer. qPCR was performed on an Applied Biosystems Quantstudio 7 Flex (Applied Biosystems, USA). Calculations for relative mRNA transcript levels were performed using the comparative CT method $\left(2^{-\Delta \Delta T}\right)$.

\section{Flow cytometric analysis}

To investigate the expression of RIPK1 and RIPK3 in SLE, intracellular RIPK production by peripheral blood leukocytes was detected by four-color flow cytometry for 50 SLE patients and 35 healthy controls. First, whole blood cells were stained with CD14, CD3, and CD19 (BD Bioscience) for 15min. Then, red blood cells were lysed. The remaining cells were then washed with PBS and fixed with fixation/permeabilization solution (BD Bioscience) for $20 \mathrm{~min}$. After fixation/permeabilization, cells were washed by BD perm/ wash buffer. Then, intracellular RIPK1/RIPK3 was stained with rabbit IgG1 anti-human RIPK1/RIPK3 


\section{Cellular Physiology Cell Physiol Biochem 2018;45:2317-2328

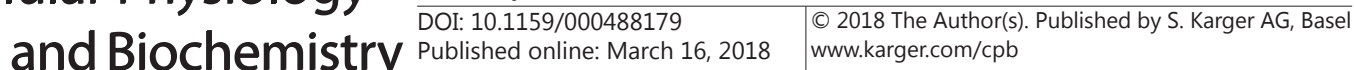 \\ Guo et al.: RIPK1 Involved in Nets Formation in SLE}

monoclonal antibody (Abcam) or control rabbit IgG1 (Abcam) by incubating for 30 min. Cells were then washed 3 times with PBS and stained with a secondary phycoerythrin (PE)-conjugated anti-rabbit IgG1 monoclonal antibody (BD Bioscience). Peripheral blood leukocytes were phenotyped by staining for CD14, CD3, and CD19 (representative markers for monocytes, T-lymphocytes, and B-lymphocytes, respectively); granulocytes were gated by cell size and granularity. Labeled cells were analyzed on an Aria flow cytometer (BD Biosciences, San Diego, CA, USA) and data were analyzed using FlowJo software (Treestar, Inc., San Carlos, CA, USA).

\section{Automatic Western Immunoblotting}

RIPK1 (1:50), RIPK3 (1:100), mixed lineage kinase domain-like (MLKL) (1:50), Fas- associated death domain-like interleukin-1b converting enzyme inhibitory protein (cFLIP) (5ug), and $\beta$-actin (1:100) were detected by automatic Simple Western analysis (Proteinsimple, USA). Isolated PBMCs and neutrophils were lysed with $100 \mu \mathrm{l}$ RIPA buffer supplemented with protease inhibitor cocktail (Roche, Mannheim, Germany). Then, cell debris was removed by centrifugation for $10 \mathrm{~min}$ at $16000 \mathrm{~g}$. The assay steps were carried out according to the instructions recommended by the manufacturer [19] and all the targeted proteins were measured simultaneously to ensure comparable data.

\section{Neutrophil isolation and stimulation}

Five milliliters of whole blood was obtained from SLE patients and healthy donors using ACD tubes (BD Vacutainer). Neutrophils were isolated immediately by a one-step gradient centrifugation method using Polymorph Prep (Axis-Shield). The mononuclear cell layer was aspirated for total RNA extraction. The neutrophil layer was isolated and resuspended in RPMI 1640 supplemented with 10\% fetal calf serum (FCS) at a density of $5 \times 10^{6}$ cells $/ \mathrm{ml}$ [10]. The preparation contained $>99 \%$ neutrophils as confirmed by flow cytometry (Aria, BD Biosciences) using anti-CD15 antibody staining (BD Bioscience). Trypan blue exclusion demonstrated that the viability was $>96 \%$ for all preparations. Neutrophils were plated in 6-well plates and allowed to settle $30 \mathrm{~min}$ in a $5 \%$ carbon dioxide atmosphere at $37^{\circ} \mathrm{C}$. Neutrophils were then incubated with Nec-1 (Sigma-Aldrich, $50 \mu \mathrm{M}$ [10]) 30 minutes before being incubated with phorbol 12-myristate 13-acetate (PMA; $10 \mathrm{ng} / \mathrm{ml}$, Sigma) for $3 \mathrm{~h}$ [20]. After 3 hours of stimulation, cells were spun down and cellfree supernatant was collected. Supernatants were stored at $-20^{\circ} \mathrm{C}$ until used. Cell death was detected by incubation with $0.1 \mu \mathrm{M}$ Sytox green dye (Life Technologies) for $10 \mathrm{~min}$.

\section{Quantification of released dsDNA in culture supernatants}

PicoGreen (Invitrogen), a fluorescent DNA dye, was added to cell-free supernatants, and the released DNA content was quantified by fluorescence spectrometry. The relative fluorescence was read with a fluorometer with a filter setting of $480 \mathrm{~nm} / 520 \mathrm{~nm}$ excitation/emission (GEN5, Bio-Tek).

\section{Confocal laser scanning for NET formation}

SLE and healthy donor neutrophils were stimulated with PMA (10 ng/ml) as described above, and seeded onto $0.001 \%$ poly-L-lysine-covered slides. Cell membranes were stained with calcein green AM (Invitrogen). For DNA labeling, DAPI (Invitrogen) was used. Neutrophil elastase (1:70) (Abcam) was incubated overnight at $4^{\circ} \mathrm{C}$ followed by washing and incubation with a secondary phycoerythrin (PE)conjugated anti-rabbit IgG1 monoclonal antibody (BD Bioscience). Slides were mounted in ProLong Gold anti-fade media (Molecular Probes) and examined with a Carl Zeiss confocal microscope.

\section{Statistical analysis}

The continuous variables were presented as mean \pm standard deviation and were analyzed by Student's t test or the Mann-Whitney U-test as appropriate. Pearson's correlation was performed where indicated. $\mathrm{P}<0.05$ was considered as statistically significant. All analyses were performed using SPSS software version13.0 (SPSSInc.; Chicago, IL, USA). 


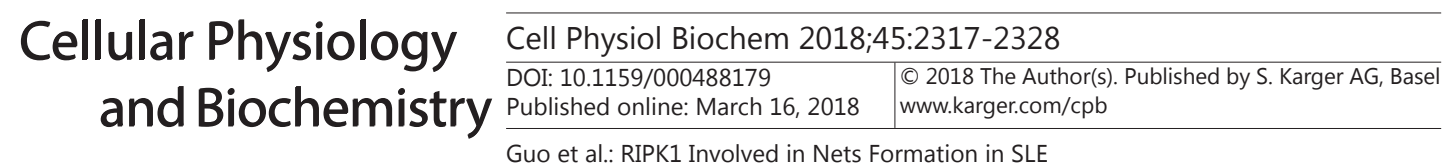

\section{Results}

Analysis of cytokine production by SLE patients and healthy controls

Cytokines levels were determined from the serum of SLE patients and healthy donors (Fig. 1A-G). The expression levels of IL-1 $\beta$, IL-8, IL-18, and IFN $\gamma$ were significantly higher in SLE patients than in healthy controls (Fig. 1A-D) $(P<0.05)$. However, some of the cytokines, like IL-2, TNF- $\alpha$, and RIPK1, were significantly lower than in healthy controls (Fig. 1E-G) (P $<0.05$ ).

\section{mRNA expression of RIPK1 and RIPK3 in SLE}

The mRNA expression of RIPK1 and RIPK3 in PBMCs from SLE patients and healthy subjects was measured by qPCR. Representative levels of RIPK1 and RIPK3 are shown in Fig. 2. The expression of RIPK1 and RIPK3 are lower in SLE patients compared to that of healthy subjects.

\section{RIPK1 and RIPK3 levels in peripheral blood leukocytes}

This is the first report to measure intracellular RIPK1 and RIPK3 expression by flow cytometry. We demonstrate that the level of RIPK1 in T-lymphocytes, B-lymphocytes, and neutrophils from patients with SLE is significantly lower than in healthy controls $(\mathrm{P}<0.05$,

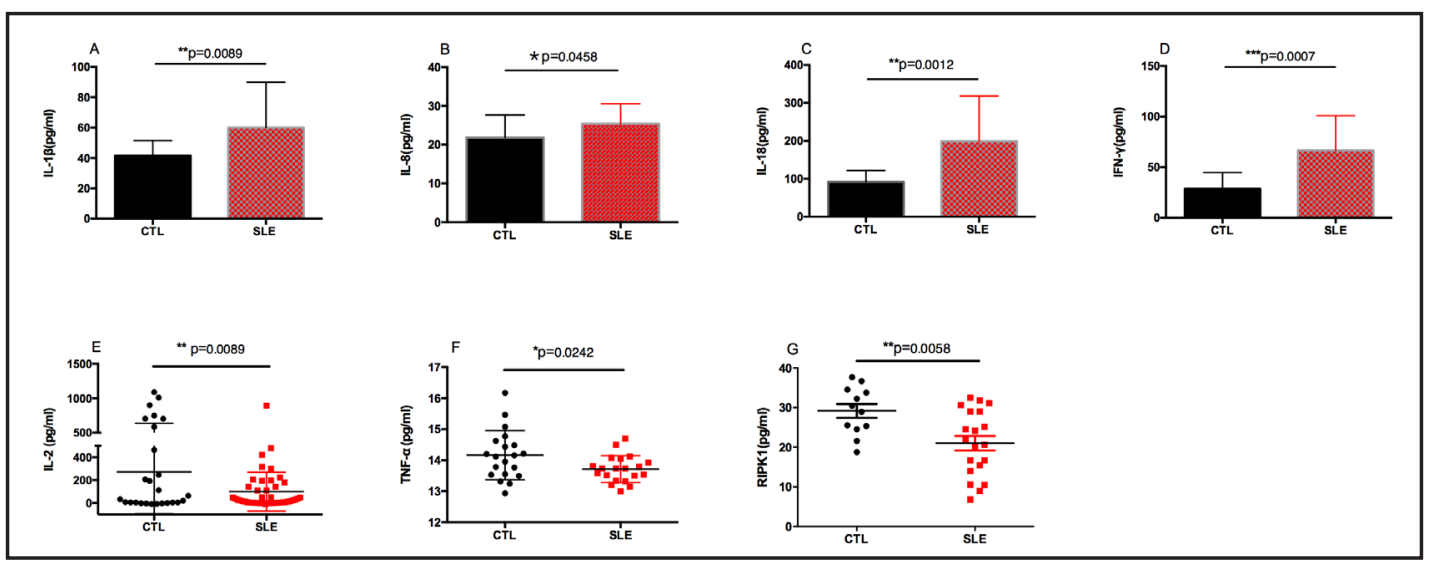

Fig. 1. Cytokines in serum of SLE patients and healthy controls. A-D, Elevation of IL-1 $\beta$ ( $n(S L E)=20$, n $(\mathrm{CTL})=20), \mathrm{IL}-8(\mathrm{n}(\mathrm{SLE})=22, \mathrm{n}(\mathrm{CTL})=19), \mathrm{IL}-18(\mathrm{n}(\mathrm{SLE})=22, \mathrm{n}(\mathrm{CTL})=19)$ and IFN- $\gamma(\mathrm{n}(\mathrm{SLE})=18, \mathrm{n}$ $(C T L)=18)$ in SLE patients versus healthy donors. E-G, Debasement of IL-2 (n(SLE)=44, n (CTL)=26), TNF- $\alpha$ $(n(S L E)=20, n(C T L)=20)$ and RIPK1 $(n(S L E)=20, n(C T L)=15)$ in plasma from SLE patients versus healthy donors. Horizontal lines show the mean. CTL= health control. ${ }^{*} \mathrm{p}<0.05,{ }^{* *} \mathrm{p}<0.01,{ }^{* * *} \mathrm{p}<0.001$ versus medium control, t-test.

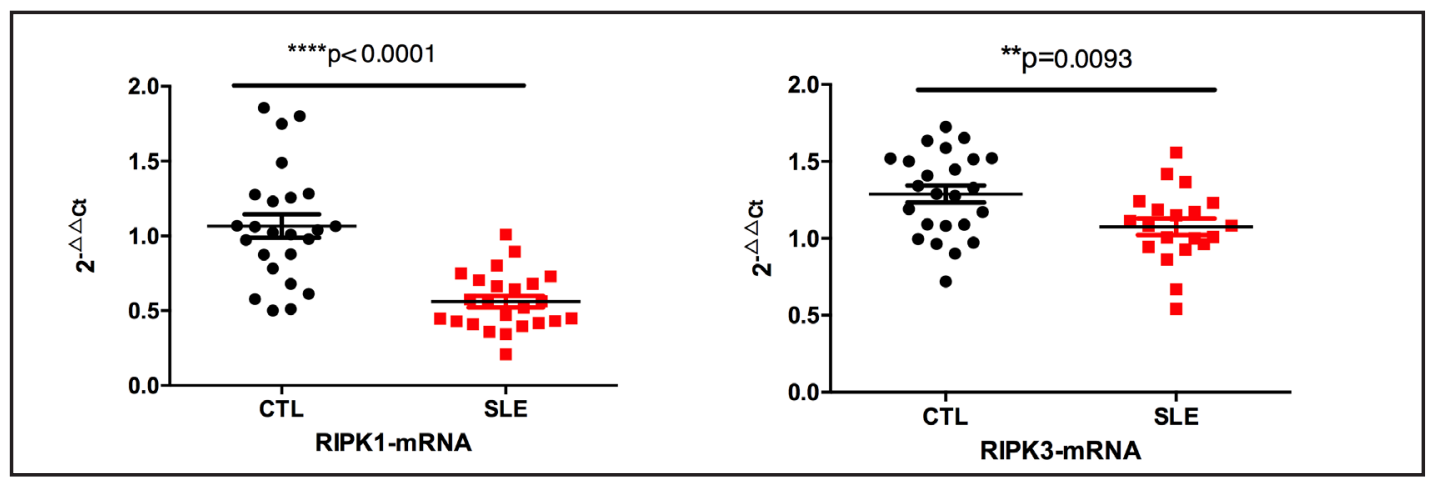

Fig. 2. The mRNA expressions of RIPK1 and RIPK3 in the PBMCs between SLE $(n=24)$ and healthy control (CTL) $(n=24)$. Horizontal lines show the mean. ${ }^{*} \mathrm{p}<0.05,{ }^{* *} \mathrm{p}<0.01,{ }^{* * *} \mathrm{p}<0.001$ versus medium control, t-test. 


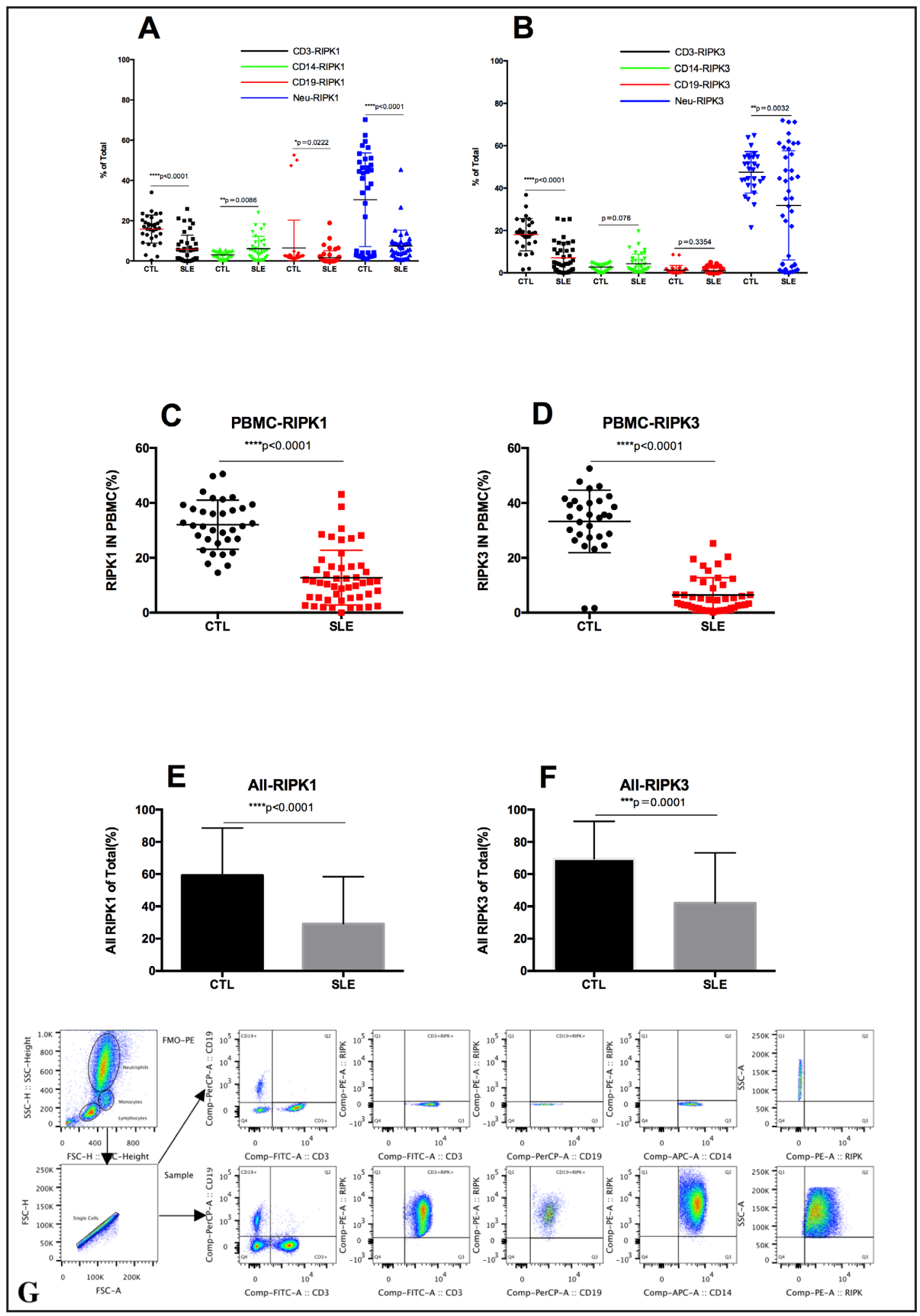

Fig. 3. The intracellular RIPK1 and RIPK3 levels in peripheral blood leukocytes by flow cytometer between SLE $(n=50)$ and CTL $(n=35)$. Horizontal lines show the mean. Fig. A-F show the total percent of RIPKs in different cell population. Fig. G shows the logic chart of flow cytometry (FMO-PE contains all the fluorescein used in the experiment, except for RIPK-PE that is being measured). All data are expressed as means \pm SD. ${ }^{*} \mathrm{p}<0.05,{ }^{* *} \mathrm{p}<0.01,{ }^{* * *} \mathrm{p}<0.001$ versus medium control, t-test. 


\section{Cellular Physiology Cell Physiol Biochem 2018;45:2317-2328 \begin{tabular}{ll|l} 
DOI: 10.1159/000488179 & a 2018 The Author(s). Published by S. Karger AG, Basel \\
www.karger.com/cpb
\end{tabular} Guo et al.: RIPK1 Involved in Nets Formation in SLE}

Fig. 3A). In addition, SLE patients had lower RIPK3 levels in T-lymphocytes and neutrophils than in healthy subjects (Fig. 3B). Furthermore, we compared the levels of RIPK1 and RIPK3 in PBMC. The levels of RIPK1 and RIPK3 were also markedly decreased in SLE patients compared to controls ( $\mathrm{P}<0.05$, Fig. 3C, D). Importantly, we reanalyzed those data in whole blood and found that total RIPK1 and RIPK3 were still significantly lower in SLE patients $(\mathrm{P}<0.05$, Fig. 3E, F).

Protein levels of RIPK1, RIPK3, and inflammation related proteins

We next analyzed RIPKs and their related proteins in cell death and inflammation signaling by automatic western immunoblotting. We detected and measured RIPK1, RIPK3, MLKL, cFLIP, and $\beta$-actin in PBMCs and neutrophils from SLE patients and healthy donors. Consistent with our flow cytometry results, RIPK1, RIPK3, and MLKL were decreased in SLE patients' PBMCs and neutrophils (Fig. 4).

Pharmacological inhibition of RIPK1 greatly enhanced NETs formation and neutrophil cell death in SLE

Neutrophils isolated from the blood of SLE patients and healthy subjects were incubated with nec-1 prior to PMA. Combined 3-color DNA (DAPI),

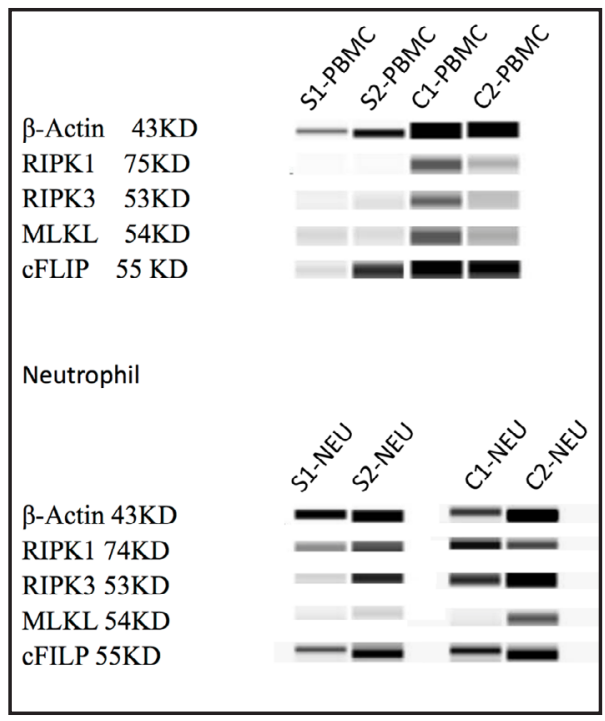

Fig. 4. RIPKs and its related proteins in cell death and inflammation signaling in PBMC and Neutrophils (NEU) of SLE patients and controls were showed above. The western blotting of each sample was running on the capillary by Wes and the ProteinSimple (a new technique) and all the samples were tested at the same time. The gray value presents the relative protein level. The experiments were totally repeated for 3 times with 2 repetitions for each group.

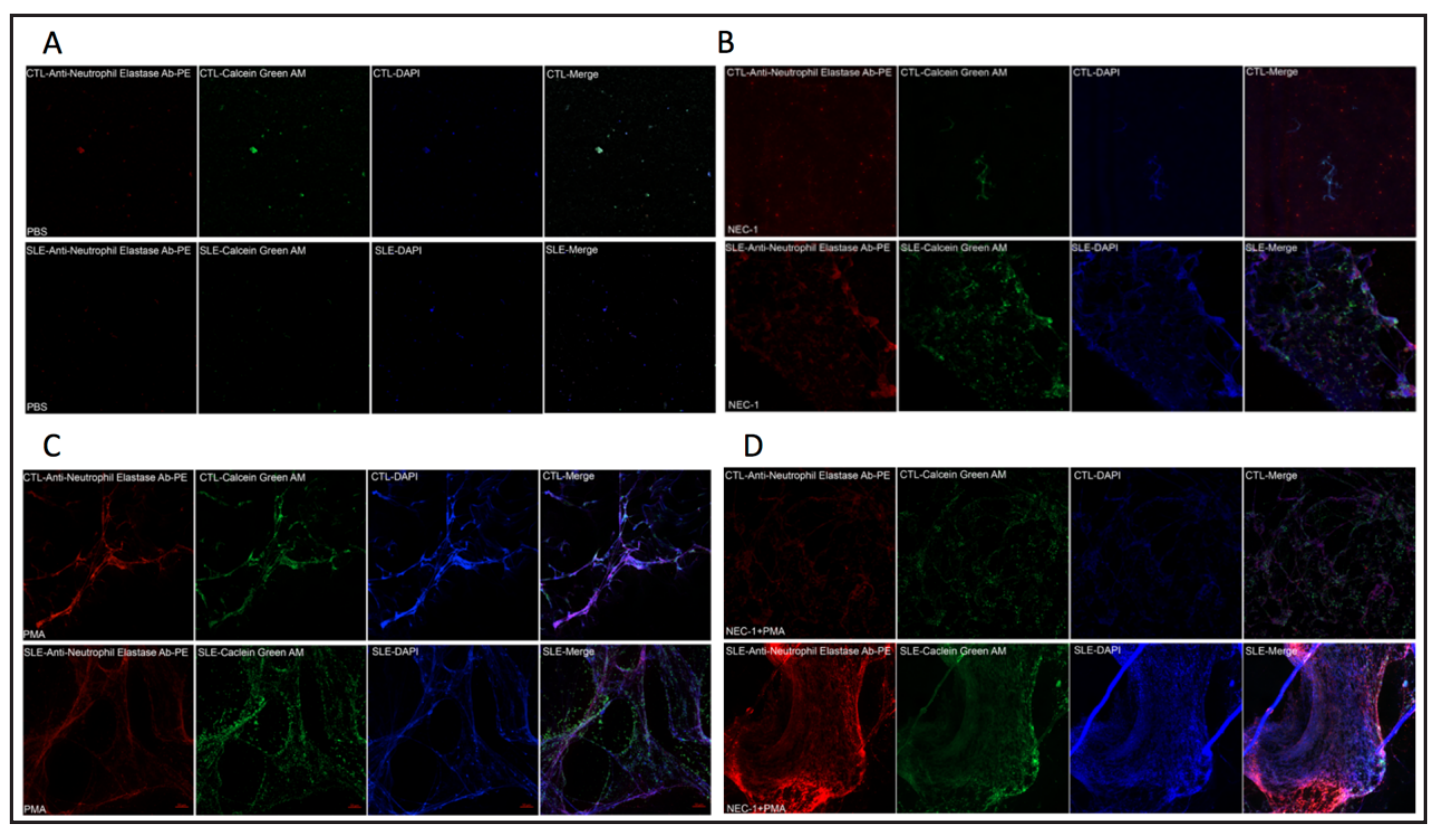

Fig. 5. Neutrophils of CTL and SLE patients were exposed to PBS (A), NEC-1 (B), PMA (C) and NEC-1+PMA (D). Confocal microscopy for the same cells stained with PE-antineurophil elastase antibody (red) and calcein green AM (green) to detect cell membrane and DAPI (blue) to detect DNA in extracellular traps. Representative images are shown at an original magnification of $10 \mathrm{x}$. 


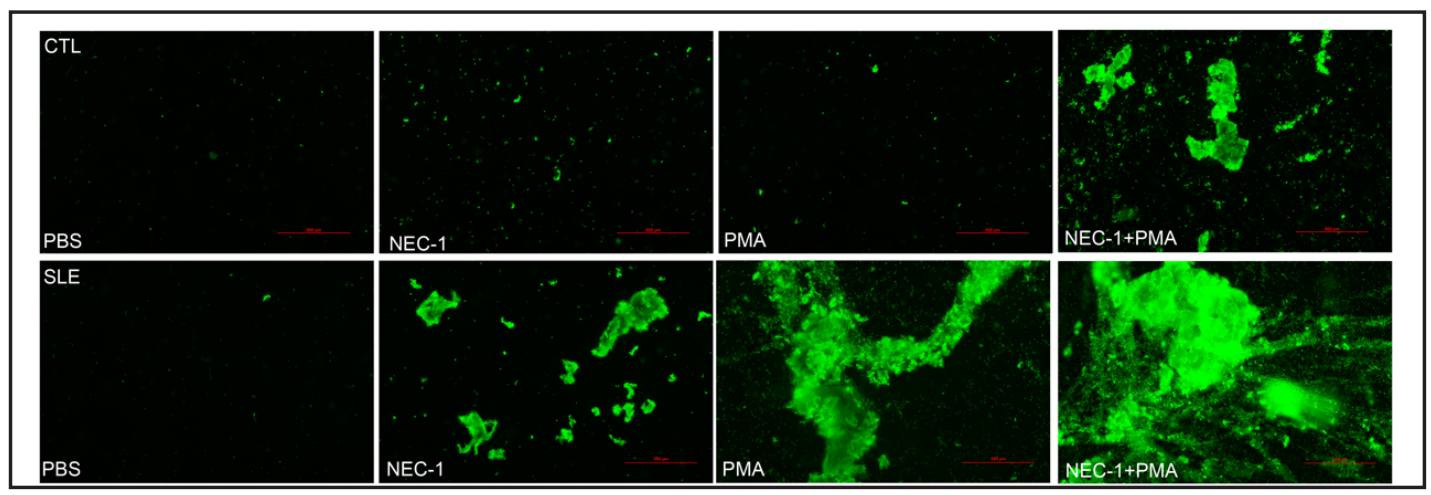

Fig. 6. Sytox green immunofluorescence $3 \mathrm{~h}$ after PMA exposure to CTL and SLE patients' neutrophils in the presence of the Nec-1 detected permeability of plasma and nuclear membranes. Different stimulus was indicated in the lower right of each picture. Representative images were shown at an original magnification of $4 \times$ (scale bar: 500 um).

Fig. 7. Neutrophils of CTL and SLE patients were exposed to NEC-1 alone or PMA in the presence of Nec-1 (50uM) as indicated below. DNA release into the supernatant was quantified by PicoGreen detection after $3 \mathrm{~h}$ as a marker of NETrelated chromatin release. NETs is shown as means \pm SE of the mean from three independent experiments. All data are expressed as means \pm SD of the mean from three independent experiments. ${ }^{*} \mathrm{p}<0.05,{ }^{* *} \mathrm{p}<0.01,{ }^{* * *} \mathrm{p}<0.001$ versus medium control, t-test.

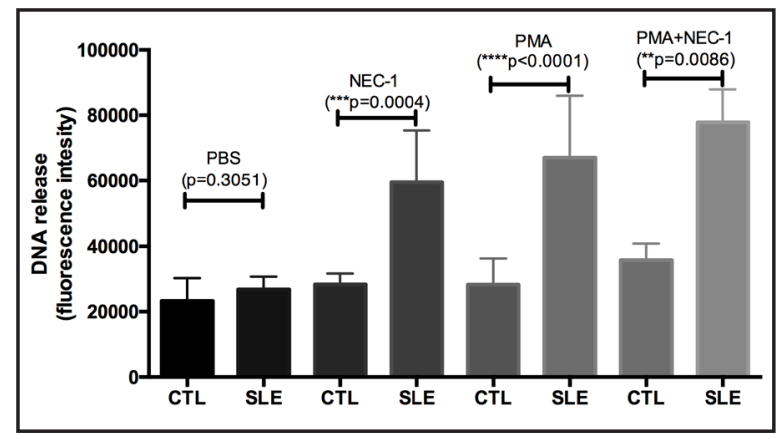

Fig. 8. Correlation of RIPK1 expression in neutrophil of SLE patients $(n=32)$ and disease activity. Fig. 8A shows a negative correlation between RIPK1 expression in whole bood and SLEDAI-2K. Fig8B-E show RIPK1 expression in neutrophils is negatively correlated with SLEDAI-2K, CRP, ESR and 24-hour total urine protein, respectively.

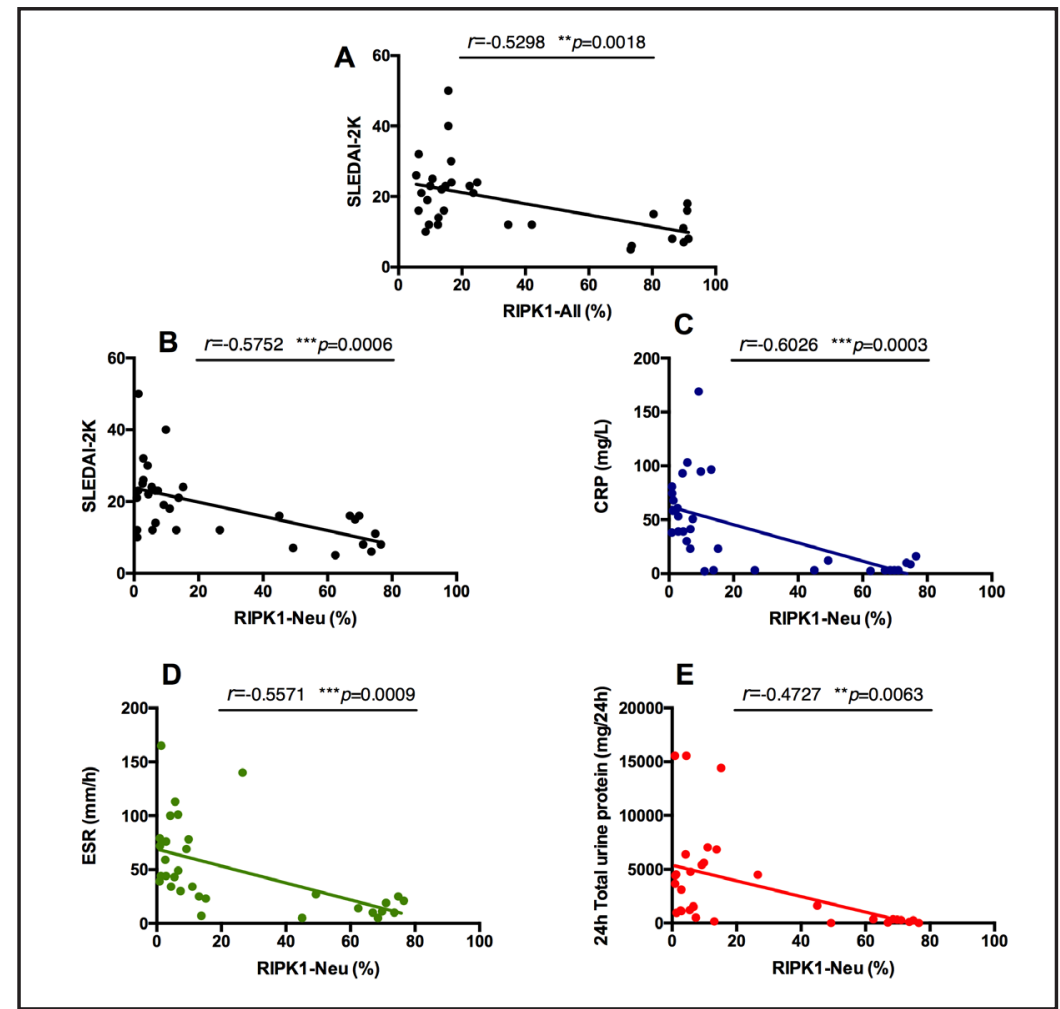




\section{Cellular Physiology Cell Physiol Biochem 2018;45:2317-2328 \\ \begin{tabular}{c|c|c|} 
DOI: 10.1159/000488179 & and Biochemistry Published online:March 16, 2018 & $\begin{array}{l}\text { O } 2018 \text { The Author(s). Published by S. Karger AG, Basel } \\
\text { www.karger.com/cpb }\end{array}$
\end{tabular} \\ Guo et al.: RIPK1 Involved in Nets Formation in SLE}

cell membrane (calcein green), and anti-neutrophil elastase antibody (PE) staining suggests that SLE neutrophils underwent extensive NETosis, as evidenced by significant web-like structure formation (Fig. 5A-D, representative images by confocal microscopy are shown at an original magnification of $10 \times$ ), when stimulated with PMA, nec-1, or a combination of the two. In parallel with apparent NETs formation, obviously increased cell death was measured by Sytox uptake (Fig. 6). Furthermore, a large release of DNA into the supernatant, assessed by Pico Green dye ( $<<0.05$, Fig. 7 ), was observed. These finding document that RIPK1 inhibitor can greatly increase NETs formation and neutrophil death in SLE patients. The experiments were repeated 3 times with 3 replicates per group.

\section{Association of RIPK1 expression with SLE disease activity}

Not surprisingly, NETs formation are associated with SLE disease activity. To further explore the role of abnormal RIPK1 expression in neutrophils in SLE, we collected 32 SLE patients' clinical information, including SLEDAI-2K, CRP, ESR, and 24-hour urine total protein. RIPK1 expression in neutrophils is negatively correlated with SLE disease activity, as determined by the SLEDAI-2K, CRP, and ESR (Fig. 8A-D). More importantly, there is also a negative correlation between levels of RIPK1 in neutrophils and 24-hour urine total protein (Fig. 8E). These results suggest that abnormal RIPK1 expression is connected to SLE disease activity, which might be a potential biomarker for the disease activity.

\section{Discussion}

In this report, we provide for the first time evidence for RIPK1 involvement in the formation of NETs in SLE patients. Furthermore, we characterize the intracellular RIPK1 and RIPK3 expression in different populations of cells, and confirmed it with automatic western blotting. Our experimental data indicate that inhibition of RIPK1 exacerbates NETs release from neutrophils treated with PMA in SLE. In addition, nec-1 treated neutrophils from SLE patients could also induce NETs formation. Furthermore, significantly increased overall cell death and large release of DNA were also observed. Based on our experimental results, we collected patients' clinical symptoms and analyzed their disease activity. Therefore, we were also able to provide evidence that RIPK1 expression in neutrophils is negatively correlated with SLE disease activity and 24-hour urine total protein.

Our data demonstrates that most proinflammatory cytokines, such as IFN- $\gamma$, IL-1 $\beta$, IL8 , and IL-18, in the serum of SLE patients are elevated compared to healthy donors. This exacerbates the patients' inflammatory status and promotes further tissue damage. Our results are consistent with previous studies indicating that those cytokines contribute to the inflammatory conditions of SLE [21, 22]. Of important note, RIPK1, IL-2, and TNF- $\alpha$ were decreased significantly. Pioneering SLE studies have demonstrated that IL-2 deficiency played an important role in the breach of immune tolerance during the pathogenesis of SLE [23]. This can be improved by low-dose IL-2 regimen. RIPK1 is on the apex of inflammatory immune responses and cell death under different conditions. The level of RIPK1 mRNA in PBMCs is significantly lower than in healthy controls. This aberrant RIPK1 expression measured by ELISA and qPCR suggest that inflammatory and cell death signals may be involved in the immunogenic injury in SLE. These findings are consistent with the studies done by Zhu et al [24]. in which they indicate that TRADD, FADD, RIPK1, and TRAF-2 in PBMCs from SLE patients are all downregulated. Honarpisheh et al [25]. report expression patterns in mice with lupus-like systemic autoimmunity, which revealed that MLKL, GPX4, and PARP1 significantly increased in the spleen with disease progression and that CASP1, RIPK1, RIPK3, and CYPD were also higher at the earlier stages but were significantly decreased in the later stages. In contrast, in the kidney, RIPK1, RIPK3, CIAP1/2, and GPX4 were significantly decreased with the progression of lupus nephritis (LN). Hence, the level of RIPK1 mRNA may predict disease progression and warrants further research.

We next investigated the RIPK1 and RIPK3 profiles in SLE patients to characterize the cell populations which are involved in the disease process. RIPK1 and RIPK3 kinases first 
emerged as mediators necroptosis [26]. However, the role of these proteins also differ in a context-dependent manner. Many pathologies involving RIPK1 and RIPK3 are associated with acute or chronic inflammation as well as disruption of immune homeostasis [12]. The contribution of RIPK1 and RIPK3 kinases to inflammatory and autoimmune pathologies has been an area of major focus and has provided many interesting conclusions $[14,27$, 28]. In this report, we demonstrate for the first time that RIPK1 levels measured by flow cytometry in T-lymphocytes, B-lymphocytes, and neutrophils from patients with SLE are significantly lower than in healthy controls. Surprisingly, RIPK1 and RIPK3 are upregulated in B cells of SLE patients treated with co-activation of R848 and anti-IgM/CD40 [29]. This data suggests that necroptosis may be the main mechanism of death in active SLE B cells. SLE patients in our study displayed lower RIPK1 and RIPK3 expression in unstimulated neutrophils compared to healthy donors. Furthermore, we performed automatic western blotting to measure related proteins in necroptosis and inflammatory signaling. Our results indicate that RIPK1, RIPK3, MLKL, and cFLIP decreased in SLE patients. Inhibition of necroptosis is mediated by a heterodimer of caspase- 8 with a long form of cFLIP, rather than the apoptosis-mediated homodimers of caspase-8 [30], suggesting that low expression of cFLIP in neutrophils in SLE might not be relevant to necroptosis. The hallmark of necroptosis is the formation of the detergent-insoluble 'necrosome' complex of RIPK1 and RIPK3 [12]. The necrosome promotes phosphorylation of a key pro-death effector, MLKL, by RIPK3. In our study, expression of RIPK1, RIPK3, and MLKL are low, specifically in neutrophils. Recent studies on proteins involved in necroptosis signaling (RIPK1, RIPK3, MLKL, cFLIP) suggest that suppression of inflammation or cell death involves RIPK1 [12, 31, 32].

In the present study, we first attempted to connect RIPK1 to the pathogenesis of SLE. SLE etiopathogenesis is a vicious cycle of autoantigen exposure, autoantibody production, chronic inflammation, and tissue damage. Recently, our department reported that NETs are important in SLE, since the release, persistent presence, and failure to remove these products represent a source of self-antigens that enhance the autoimmune and inflammatory process, leading to exacerbated tissue injury in SLE [20,33]. In addition, the current experimental approaches and pharmacological inhibition to study NETs in gout provides direct evidence that the number of NETs is diminished when PMA-stimulated neutrophils are treated with nec-1 [10]. Furthermore, the number of NETs observed was reduced by at least $50 \%$ in PMAstimulated neutrophils isolated from RIPK3 knock-out mice compared to wild-type mice [10]. Therefore, our study is the first to investigate the role of intracellular RIPK1 in PMAinduced NETs formation in SLE. We identified that stimulation with nec-1 or PMA increased overall cell death and greatly enhanced the formation of NETs in SLE patients. In contrast to a recent study [10], Desai et al. suggest that nec-1 inhibits monosodium urated crystalinduced NETs formation and gout-like tophus formation in vivo, confirming the involvement of neutrophil necroptosis in NETs release [10, 34]. However, Amini et al [35]. reported no difference in the percentage of NET-forming neutrophils between PMA-stimulated neutrophils incubated in the presence or absence of nec-1. These opposing conclusions might result from different experimental approaches [36]. It seems likely that additional intrinsic factors controlling differential activation of RIPK1-dependent responses may exist in specific disease contexts. Our data suggests that RIPK1 and RIPK3 might not only be involved in necroptosis, but also participate in the formation of NETs in SLE patients, when the conformation of RIPK1 is changed by nec-1. In $\alpha$ GalCer-induced autoimmune hepatitis, RIPK1 silencing also exacerbates injury by massive induction of apoptosis [37]. Nec-1 accelerated disease progression in both cerulein-induced pancreatitis and TNF $\alpha$-mediated shock [38]. Characterizing the exact mechanism is critical for future research.

From a clinical perspective, kinase activity of RIPK1 appears to be positioned at the apex of the regulation. The low expression of RIPKs might in part explain the activated state of neutrophils. We analyzed patients' clinical information, such as SLEDAI-2K, CRP, ESR, and $24 \mathrm{~h}$-urine total protein, to link RIPK1 expression in neutrophils of SLE patients. There is a negative correlation between levels of RIPK1 in neutrophils and 24-hour urine total protein. We also found a negative correlation between levels of RIPK1 in neutrophils and SLE disease 


\section{Cellular Physiology Cell Physiol Biochem 2018;45:2317-2328 \\ \begin{tabular}{l|l|l} 
and Biochemistry Publisned onIIne: IVIarch 16, 2018 & $\begin{array}{l}\text { (c) } 2018 \text { The Author(s). Published by S. Karger AG, Basel } \\
\text { www.karger.com/cpb }\end{array}$
\end{tabular} \\ Guo et al.: RIPK1 Involved in Nets Formation in SLE}

activity (SLEDAI-2K, CRP, and ESR). Therefore, RIPK1 might be an indicator, a surrogate marker of disease activity in SLE, and warrants further studies to validate. This preliminary study provides a stepping stone towards further characterization of RIPK1 in the formation of PMA-induced NETs in neutrophils of SLE patients.

\section{Acknowledgements}

This work was supported by the National Key Research and Development Program of China (2017YFC0909000) and the National Natural Science Foundation of China (Grant No: 81373209).

\section{Disclosure Statement}

The authors declare that there is no Disclosure Statement.

\section{References}

1 Bosch X: Systemic Lupus Erythematosus and the Neutrophil. N Engl J Med 2011;365:758-760.

2 Lee HT, Wu TH, Lin CS, Lee CS, Wei YH, Tsai CY, Chang DM: The pathogenesis of systemic lupus erythematosus - From the viewpoint of oxidative stress and mitochondrial dysfunction. Mitochondrion 2016;30:1-7.

- Podolska MJ, Biermann MH, Maueroder C, Hahn J, Herrmann M: Inflammatory etiopathogenesis of systemic lupus erythematosus: an update. J Inflamm Res 2015;8:161-171.

4 Kaplan MJ: Neutrophils in the pathogenesis and manifestations of SLE. Nat Rev Rheumatol 2011;7:691699.

-5 Galluzzi L, Vitale I, Abrams JM, Alnemri ES, Baehrecke EH, Blagosklonny MV, Dawson TM, Dawson VL, El-Deiry WS, Fulda S, Gottlieb E, Green DR, Hengartner MO, Kepp O, Knight RA, Kumar S, Lipton SA, Lu X, Madeo F, Malorni W, Mehlen P, Nuñez G, Peter ME, Piacentini M, Rubinsztein DC, Shi Y, Simon HU, Vandenabeele P, White E, Yuan J, Zhivotovsky B, Melino G, Kroemer G: Molecular definitions of cell death subroutines: recommendations of the Nomenclature Committee on Cell Death 2012 Cell Death Differ 2012;19:107-120.

6 Delgado-Rizo V, Martínez-Guzmán MA, Iñiguez-Gutierrez L, García-Orozco A, Alvarado-Navarro A, FafutisMorris M: Neutrophil Extracellular Traps and Its Implications in Inflammation: An Overview. Frontiers in Immunology 2017;8:81.

7 Pires RH, Felix SB, Delcea M: The architecture of neutrophil extracellular traps investigated by atomic force microscopy: Nanoscale 2016;8:14193-14202.

8 Mahajan A, Herrmann M, Munoz LZ: Clearance De ciency and Cell Death Pathways: A Model for the Pathogenesis of SLE. Front Immunol 2016;7:35.

G Grayson PC, Kaplan MJ: At the Bench: Neutrophil extracellular traps (NETs) highlight novel aspects of innate immune system involvement in autoimmune diseases. J Leukoc Biol 2016;99:253-264.

10 Desai J, Kumar SV, Mulay SR, Konrad L, Romoli S, Schauer C, Herrmann M, Bilyy R, Müller S, Popper B, Nakazawa D, Weidenbusch M, Thomasova D, Krautwald S, Linkermann A, Anders HJ: PMA and crystalinduced neutrophil extracellular trap formation involves RIPK1-RIPK3-MLKL signaling. Eur J Immunol 2016;46:223-229.

-11 Zhou W, Yuan J: Necroptosis in health and diseases. Semin Cell Dev Biol 2014;35:14-23.

12 Wegner KW, Saleh D, Degterev A: Complex Pathologic Roles of RIPK1 and RIPK3: Moving Beyond Necroptosis. Trends Pharmacol Sci 2017;38:202-225.

-13 Orozco S, Oberst A: RIPK3 in cell death and inflammation: the good, the bad, and the ugly. Immunol Rev 2017;277:102-112.

14 Jin L, Chen J, Liu XY, Jiang CC, Zhang XD: The double life of RIPK1. Mol Cell Oncol 2016;3:e1035690.

15 Wang FF, Zhu LA, Zou YQ, Zheng H, Wilson A, Yang CD, Shen N, Wallace DJ, Weisman MH, Chen SL, Lu LJ: New insights into the role and mechanism of macrophage migration inhibitory factor in steroid-resistant patients with systemic lupus erythematosus. Arthritis Res Ther 2012;14:R103.

16 Ofengeim D, Yuan J: Regulation of RIP1 kinase signalling at the crossroads of inflammation and cell death. Nat Rev Mol Cell Biol 2013;14:727-736. 


\section{Cellular Physiology Cell Physiol Biochem 2018;45:2317-2328 \begin{tabular}{ll|l} 
DOI: 10.1159/000488179 & Ond Biochemistry \\
Publisned onlne: IVarch 16, 2018 & $\begin{array}{l}\text { 2018 The Author(s). Published by S. Karger AG, Basel } \\
\text { www.karger.com/cpb }\end{array}$ \\
\cline { 2 - 3 }
\end{tabular}

17 Feletar M, Ibanez D, Urowitz MB, Gladman DD: The impact of the 1997 update of the American College of Rheumatology revised criteria for the classification of systemic lupus erythematosus: what has been changed? Arthritis Rheum 2003;48:2067-2069.

18 Rao V, Gordon C: Advances in the assessment of lupus disease activity and damage. Curr Opin Rheumatol 2014;26:510-519.

19 Harris VM: Protein detection by Simple Western analysis. Methods Mol Biol 2015;1312:465-468.

20 Wang H, Li T, Chen S, Gu Y, Ye S: Neutrophil Extracellular Trap Mitochondrial DNA and Its Autoantibody in Systemic Lupus Erythematosus and a Proof-of-Concept Trial of Metformin. Arthritis Rheumatol 2015;67:3190-3200.

21 Wu Y, Cai B, Zhang J, Shen B, Huang Z, Tan C, Baan CC, Wang L: IL-1beta and IL-6 Are Highly Expressed in RF+IgE+ Systemic Lupus Erythematous Subtype. J Immunol Res 2017;2017:5096741.

22 Wu CY, Yang HY, Yao TC, Liu SH, Huang JL: Serum IL-18 as biomarker in predicting long-term renal outcome among pediatric-onset systemic lupus erythematosus patients. Medicine (Baltimore) 2016;95:e5037.

23 Humrich JY, Riemekasten G: Clinical trials: The rise of IL-2 therapy - a novel biologic treatment for SLE. Nat Rev Rheumatol 2016;12:695-696.

24 Zhu L, Yang X, Chen W, Li X, Ji Y, Mao H, Nie J, Yu X: Decreased expressions of the TNF-alpha signaling adapters in peripheral blood mononuclear cells (PBMCs) are correlated with disease activity in patients with systemic lupus erythematosus. Clin Rheumatol 2007;26:1481-1489.

25 Honarpisheh M, Desai J, Marschner JA, Weidenbusch M, Lech M, Vielhauer V, Anders HJ, Mulay SR: Regulated necrosis-related molecule mRNA expression in humans and mice and in murine acute tissue injury and systemic autoimmunity leading to progressive organ damage, and progressive fibrosis. Biosci Rep 2016;36:pii:e00425.

26 Green DR, Oberst A, Dillon CP, Weinlich R, Salvesen GS: RIPK-dependent necrosis and its regulation by caspases: a mystery in five acts. Mol Cell 2011;44:9-16.

27 Saleh D, Najjar M, Zelic M, Shah S, Nogusa S, Polykratis A, Paczosa MK, Gough PJ, Bertin J, Whalen M, Fitzgerald KA, Slavov N, Pasparakis M, Balachandran S, Kelliher M, Mecsas J, Degterev A: Kinase Activities of RIPK1 and RIPK3 Can Direct IFN-beta Synthesis Induced by Lipopolysaccharide. J Immunol 2017;198:4435-4447.

28 Ito Y, Ofengeim D, Najafov A, Das S, Saberi S, Li Y, Hitomi J, Zhu H, Chen H, Mayo L, Geng J, Amin P, DeWitt JP, Mookhtiar AK, Florez M, Ouchida AT, Fan JB, Pasparakis M, Kelliher MA, Ravits J, Yuan J: RIPK1 mediates axonal degeneration by promoting inflammation and necroptosis in ALS. Science 2016;353:603-608.

29 Fan H, Liu F, Dong G, Ren D, Xu Y, Dou J, Wang T, Sun L, Hou Y: Activation-induced necroptosis contributes to B-cell lymphopenia in active systemic lupus erythematosus. Cell Death Dis 2014;5:e1416.

30 Dillon Christopher P, Oberst A, Weinlich R, Janke LJ, Kang TB, Ben-Moshe T, Mak TW, Wallach D, Green DR: Survival Function of the FADD-CASPASE-8-cFLIP (L) Complex. Cell Reports 2012;1:401-407.

-31 Dillon CP, Oberst A, Weinlich R, Janke LJ, Kang TB, Ben-Moshe T, Mak TW, Wallach D, Green DR: RIPK1 blocks early postnatal lethality mediated by caspase-8 and RIPK3. Cell 2014;157:1189-1202.

-32 Lin J, Kumari S, Kim C, Van TM, Wachsmuth L, Polykratis A, Pasparakis M: RIPK1 counteracts ZBP1mediated necroptosis to inhibit inflammation. Nature 2016;540:124-128.

-33 Vidal Delgado-Rizo, Marco A Martinez-Guzman, Liliana Iñiguez-Gutierrez, Alejandra García-Orozco, Anabell Alvarado-Navarro, Mary Fafutis-Morris: Neutrophil extracellular Traps and its implications in in ammation: An Overview. Front Immunol 2017;8:81.

34 Desai J, Mulay SR, Nakazawa D, Anders HJ: Matters of life and death. How neutrophils die or survive along NET release and is "NETosis" = necroptosis? Cell Mol Life Sci 2016;73:2211-2219.

-35 Amini P, Stojkov D, Wang X, Wicki S, Kaufmann T, Wong WW, Simon HU, Yousefi S: NET formation can occur independently of RIPK3 and MLKL signaling. Eur J Immunol 2016;46:178-184.

-36 Naccache PH, Fernandes MJ: Challenges in the characterization of neutrophil extracellular traps: The truth is in the details. Eur J Immunol 2016;46:52-55.

37 Suda J, Dara L, Yang L, Aghajan M, Song Y, Kaplowitz N, Liu ZX: Knockdown of RIPK1 Markedly Exacerbates Murine Immune-Mediated Liver Injury through Massive Apoptosis of Hepatocytes, Independent of Necroptosis and Inhibition of NF-kappaB. J Immunol 2016;197:3120-3129.

38 Linkermann A, Bräsen JH, De Zen F, Weinlich R, Schwendener RA, Green DR, Kunzendorf U, Krautwald S: Dichotomy between RIP1- and RIP3-mediated necroptosis in tumor necrosis factor-alpha-induced shock. Mol Med 2012;18:577-586. 